\title{
Autologous CMV-specific T cells are a safe adjuvant immunotherapy for primary glioblastoma multiforme
}

\author{
Corey Smith, ${ }^{1}$ Katie E. Lineburg, ${ }^{1}$ J. Paulo Martins, ${ }^{1}$ George R. Ambalathingal, ${ }^{1}$ Michelle A. Neller, ${ }^{1}$ Beth Morrison, ${ }^{2}$ \\ Katherine K. Matthews, ${ }^{1}$ Sweera Rehan, ${ }^{1}$ Pauline Crooks, ${ }^{1}$ Archana Panikkar, ${ }^{1}$ Leone Beagley, ${ }^{1}$ Laetitia Le Texier, ${ }^{1}$ \\ Sriganesh Srihari, ${ }^{1}$ David Walker, ${ }^{2}$ and Rajiv Khanna'
}

'QIMR Berghofer Centre for Immunotherapy and Vaccine Development and Tumour Immunology Laboratory, Department of Immunology, QIMR Berghofer Medical Research Institute, Brisbane, Queensland, Australia. ${ }^{2}$ NEWRO Foundation, Brisbane, Queensland, Australia.

\begin{abstract}
BACKCROUND. The recent failure of checkpoint-blockade therapies for glioblastoma multiforme (GBM) in late-phase clinical trials has directed interest toward adoptive cellular therapies (ACTs). In this open-label, first-in-human trial, we have assessed the safety and therapeutic potential of cytomegalovirus-specific (CMV-specific) ACT in an adjuvant setting for patients with primary CBM, with an ultimate goal to prevent or delay recurrence and prolong overall survival.
\end{abstract}

\begin{abstract}
METHODS. Twenty-eight patients with primary GBM were recruited to this prospective study, $\mathbf{2 5}$ of whom were treated with in vitro-expanded autologous CMV-specific T cells. Participants were monitored for safety, progression-free survival, overall survival (OS), and immune reconstitution.

RESULTS. No participants showed evidence of ACT-related toxicities. Of $\mathbf{2 5}$ evaluable participants, 10 were alive at the completion of follow-up, while 5 were disease free. Reconstitution of CMV-specific T cell immunity was evident and CMVspecific ACT may trigger a bystander effect leading to additional T cell responses to nonviral tumor-associated antigens through epitope spreading. Long-term follow-up of participants treated before recurrence showed significantly improved OS when compared with those who progressed before ACT (median 23 months, range 7-65 vs. median 14 months, range 5-19; $P=0.018$ ). Gene expression analysis of the ACT products indicated that a favorable T cell gene signature was associated with improved long-term survival.
\end{abstract}

CONCLUSION. Data presented in this study demonstrate that CMV-specific ACT can be safely used as an adjuvant therapy for primary CBM and, if offered before recurrence, this therapy may improve OS of CBM patients.

TRIAL REGISTRATION. anzctr.org.au: ACTRN12615000656538.

FUNDING. Philanthropic funding and the National Health and Medical Research Council (Australia).

\section{Introduction}

Therapeutic intervention with cytotoxic T lymphocytes is emerging as a powerful tool for the induction of sustained immune control of malignancies and virus-associated complications (1). Adoptive cellular therapy (ACT) utilizing genetically modified or nonmodified antigen-specific $\mathrm{T}$ cells has provided sustained clinical responses in certain clinical settings $(2,3)$. To date, the therapeutic benefit of ACT has been limited in solid tumors, most likely due to the immune microenvironment and the impact of advanced tumor burden, which is associated with

Conflict of interest: CS and RK hold international patents (US7976845B2, US7524503B2, US20180207261A1, and AU2017271136A1) on CMV vaccines and immunotherapy. RK and CS act as consultants for Atara Biotherapeutics. RK is on the Scientific Advisory Board of Atara Biotherapeutics.

Copyright: (5) 2020, American Society for Clinical Investigation.

Submitted: March 31, 2020; Accepted: July 29, 2020; Published: October 12, 2020

Reference information: J Clin Invest. 2020;130(11):6041-6053.

https://doi.org/10.1172/JCl138649. rapid progression and poor overall survival (OS) (4). In this context, early intervention with immunotherapy following primary diagnosis and first-line treatment offers an attractive alternative to treatment in advanced stages of disease (5). The additional advantage of preemptive therapeutic intervention with ACT, after primary diagnosis, is the reduced $\mathrm{T}$ cell-to-target antigen ratio, which should limit the cellular dysfunction associated with chronic T cell activation in the setting of high tumor burden (6).

Despite great success in many aspects of tumor therapy over the past 5 decades, including recent advances in antibody and cellular immunotherapies, dramatically improved outcomes for patients with glioblastoma multiforme (GBM) remain elusive $(7,8)$. Molecularly targeted therapies and checkpoint inhibitors have thus far failed to offer significant survival benefit beyond the current median OS of 14 to 16 months after initial diagnosis (9-12). As such, the standard of care for patients with GBM remains largely unchanged from surgical resection followed by radiotherapy and chemotherapy with temozolomide. However, evidence of prolonged survival in a subset of patients following either checkpoint inhibition or ACT 


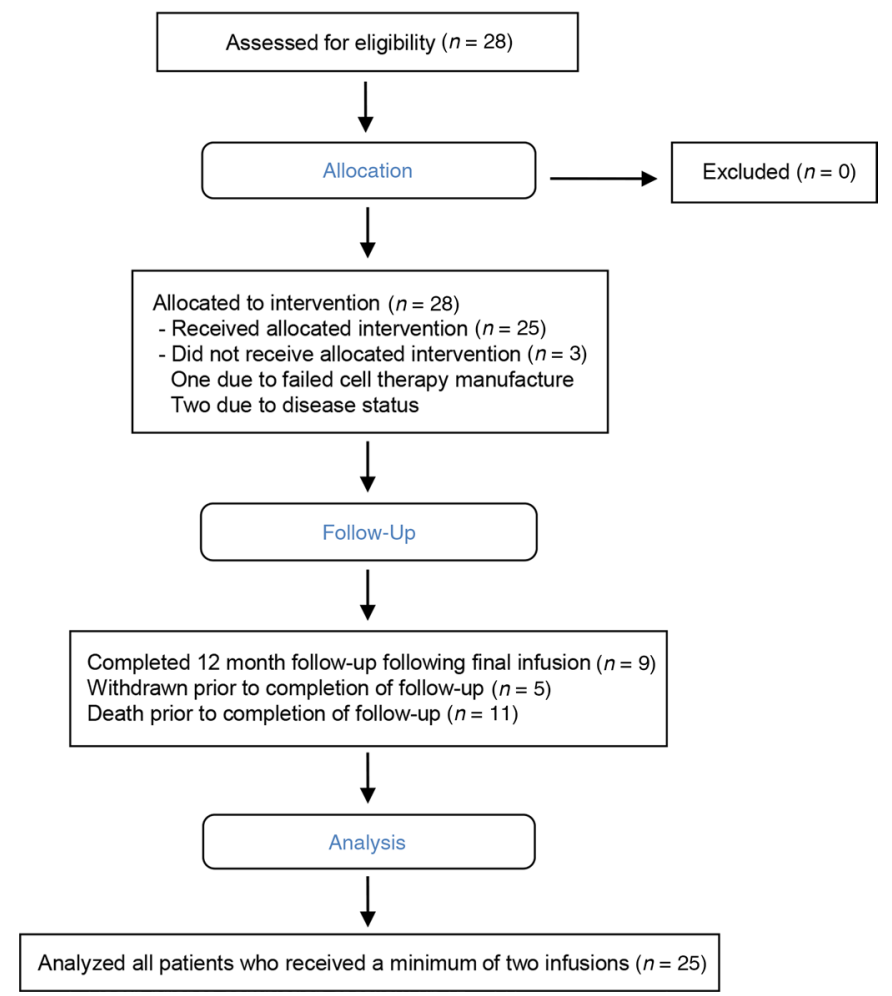

Figure 1. CONSORT diagram showing participant allocation, follow-up, and analysis.

provides cause for optimism about the impact of immunotherapy on patient outcomes in the GBM setting (13-17). Considering the aggressive nature of GBM, the high rate of recurrent disease, and the overall poor survival outcomes for these patients, identifying the most appropriate strategies for administering immunotherapy to treat GBM is paramount to its success. We previously completed a phase I clinical trial assessing the safety and potential efficacy of using cytomegalovirus-specific (CMV-specific) ACT to treat patients with recurrent GBM (18). Although the role of CMV in GBM remains controversial (19), the presence of foreign CMV antigens within GBM tissue makes this an attractive target for ACT (20-23). Our initial studies using CMV-specific ACT against recurrent GBM provided encouraging clinical responses, with multiple patients showing prolonged OS (24). To assess whether earlier intervention with ACT improved patient outcomes, we initiated a phase I safety study utilizing CMV-specific ACT to treat GBM following primary resection and chemo/radiotherapy. Here the preemptive administration of CMV-specific ACT before evidence of recurrence was safe and provided encouraging clinical evidence of improved progression-free survival (PFS) and OS compared with the initiation of ACT following disease progression. Furthermore, these patients showed clear evidence of improved CMV-specific $\mathrm{T}$ cell immunity following ACT.

\section{Results}

Patient characteristics and ACT. Twenty-eight patients diagnosed with primary GBM were recruited for this study (Figure 1, Tables 1 and 2, and Supplemental Table 1; supplemental material available online with this article; https://doi.org/10.1172/

\section{Table 1. Clinical characteristics of CBM patients enrolled in the study}

$\begin{array}{lc}\text { Nume } & 28 \\ \text { Age of patients } & \begin{array}{c}\text { Median: } 59 \\ \text { (range: } 32-74)\end{array} \\ \text { Sex } & 10 \\ \text { Female } & 18 \\ \text { Male } & \\ \text { GBM location } & 9^{A} \\ \text { Frontal } & 7^{A} \\ \text { Temporal } & 5 \\ \text { Parietal } & 4 \\ \text { Parieto-occipital } & 2 \\ \text { Occipital } & 1 \\ \text { Parieto-temporal } & 1 \\ \text { Thalamic } & 3 \text { of } 28 \\ \text { IDH mutation } & 11 \text { of } 28 \\ \text { Methylated } \text { MGMT } & 28 \text { of } 28 \\ \text { Pre-ACT CTx/RT } & 21 \text { of } 25 \\ \text { During ACT CTx } & 16 \text { of } 25 \\ \text { Post-ACT CTx } & \\ \text { AOne patient described as both temporal and frontal. CTx, chemotherapy; } \\ \text { RT, radiotherapy. }\end{array}$

JCI138649DS1). No patient had evidence of progression before recruitment. This cohort was $64 \%$ male and $36 \%$ female, with a median age of 59 years. Patients were diagnosed with primary GBM, predominantly in the frontal and temporal regions; $11 \%$ of patients had isocitrate dehydrogenase (IDH) mutations and $39 \%$ had methylation of the methylguanine-DNA methyltransferase gene (MGMT) (Table 1).

CMV-specific T cells for ACT were successfully generated from 27 of 28 patients. ACT products contained a median of $79.3 \%$ (range $12.0 \%-97.3 \%$ ) $\mathrm{CD}^{+} \mathrm{CD}^{+} \mathrm{T}$ cells and $12.8 \%$ (range $2.4 \%-80.2 \%$ ) $\mathrm{CD}^{+} \mathrm{CD}^{+} \mathrm{T}$ cells (Figure $2 \mathrm{~A}$ ). The remaining cells were predominantly natural killer cells. Evidence of CMV-specific reactivity was detected in all ACT products (Figure 2B), with a median percentage of $40 \%$ CMV-specific IFN- $\gamma$-producing cells. Patient 2105-12's ACT product displayed low CMV reactivity and failed to meet manufacturing criteria due to low cell yield. CMV reactivity in 25 of the remaining 27 ACT products was dominated by $\mathrm{CD}^{+} \mathrm{T}$ cells, while ACT products from patients 2105-02 and 2105-27 were dominated by CMV-specific CD4 ${ }^{+} \mathrm{T}$ cells (Table 3 and Figure 2B). ACT products displayed reactivity against a median of $6 \mathrm{CMV}-$ encoded peptide epitopes (range 1-9) restricted by a median of 3 different HLA types (range 1-7). Patient HLA types and the CMV epitopes encoded in the peptide pool are listed in Supplemental Tables 1 and 2. Three patients, including 2105-12, did not receive ACT. The additional 2 patients, 2105-07 and 2105-19, were withdrawn before ACT commencement due to advanced disease. The remaining 25 patients received a minimum of 2 and maximum of 6 doses of ACT (median 5.5), depending on 


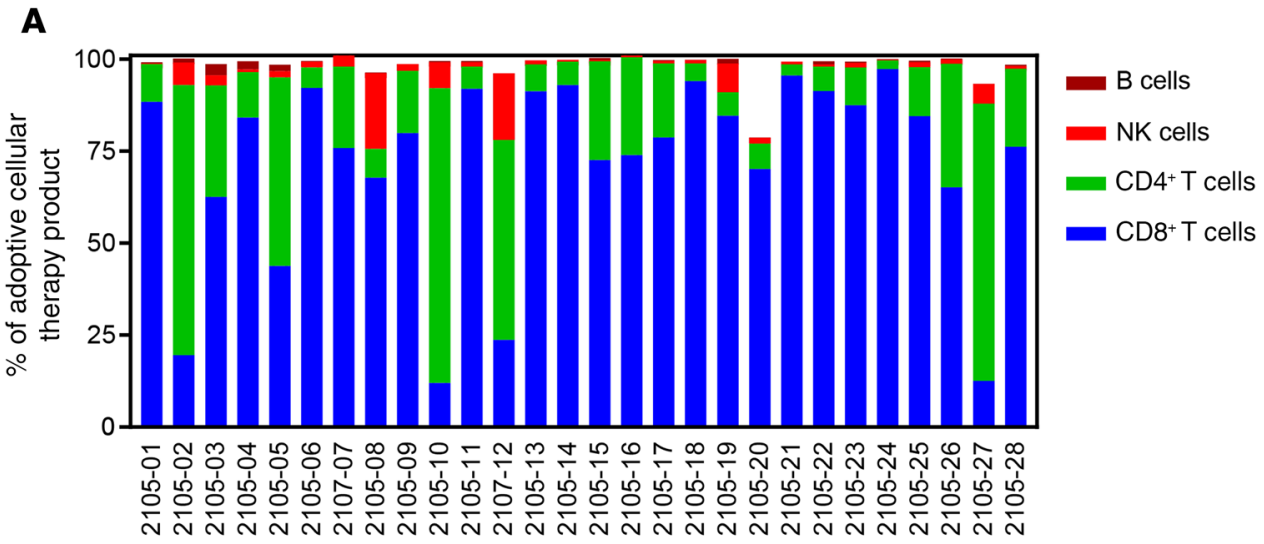

B

Patient code

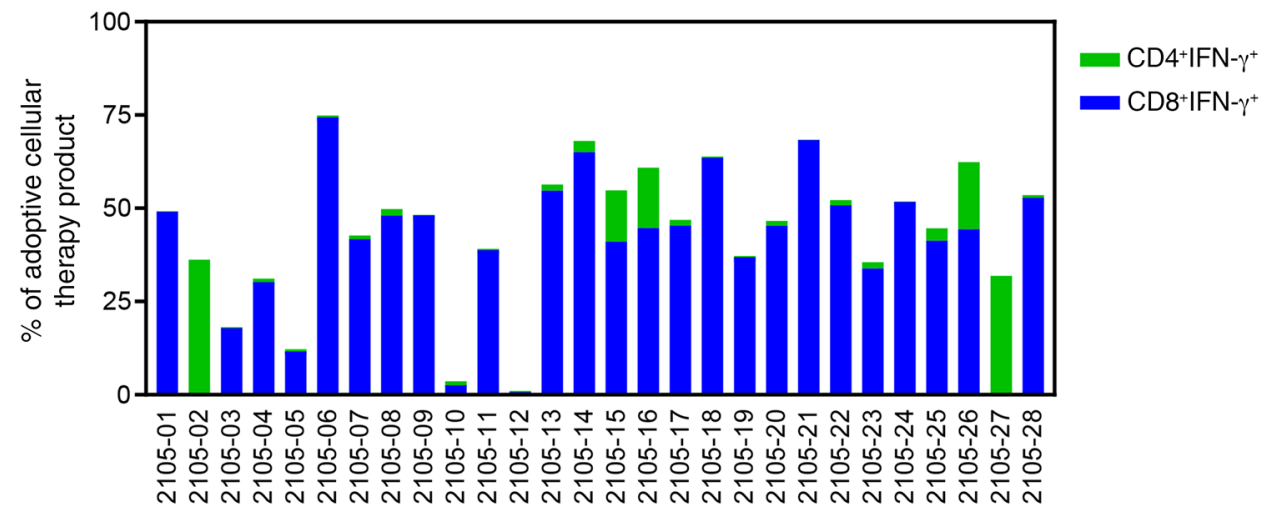

Patient code

Figure 2. Characteristics of CMV-specific ACT products generated for GBM adoptive immunotherapy. (A) The phenotypic characteristics of T cells were assessed using standard TBNK analysis. Data represent the proportion of CD4+ $T$ cells, CD8 ${ }^{+} T$ cells, NK cells, and B cells in the final products. (B) T cells were assessed for the intracellular production of IFN- $\gamma$ following recall with the CMV peptide pool used to generate the cell therapy. Data represent CD8 or $\mathrm{CD}^{+} \mathrm{T}$ cells producing IFN- $\gamma$ as a proportion of total viable lymphocytes.

the availability of product, at $2 \times 10^{7}$ cells $/ \mathrm{m}^{2}$. Patient $2105-25$ was withdrawn following 2 doses due to advanced disease. The total ACT dose administered to each patient is provided in Table 3. Twenty of the 25 treated patients commenced ACT infusions before evidence of disease progression, while the remaining 5 patients progressed before the first ACT infusion.

Safety profile and clinical responses to $C M V$-specific ACT in primary GBM. Prior to recruitment, all patients underwent surgical resection of their primary tumor and had no evidence of relapse. Patients then received standard radiotherapy and chemotherapy, and ACT commenced at a median of 162 days and following diagnosis 56 days after recruitment. CMV-specific ACT was well tolerated in all patients. Adverse events were recorded throughout the trial, with terms and grades assigned using the Common Terminology Criteria for Adverse Events v4.0. Their association with ACT was classified using a 5-point scale: unrelated, unlikely to be related, possibly related, probably related, or definitely related. The majority of adverse events were determined to be related to the natural history of GBM and therefore unrelated to ACT. In addition, no adverse events that were possibly, probably, or definitely associated with ACT were observed. A summary of adverse events that were unlikely to be related to therapy are provided in Table 4. Patients were monitored for disease progression using magnetic resonance imaging (MRI) during the course of ACT and for 12 months following their final infusion. Representative MRI scans from 4 patients are shown in Figure 3. Of 25 evaluable patients, 5 were withdrawn before completion of follow-up, 5 showed no evidence of tumor progression, and 10 were alive at completion of follow-up (Figure 4 and Table 3). Twelve months after completion of ACT, the PFS and OS of all treated patients were $20 \%$ and $52 \%$, respectively. Furthermore, PFS and OS of patients who were offered CMV-specific ACT as adjuvant therapy were $25 \%$ and $60 \%$, respectively.

Immunological monitoring following CMV-specific ACT. To assess the impact ACT has on CMV-specific cellular immunity in patients with GBM, peripheral blood mononuclear cells (PBMCs) were assessed for $\mathrm{CMV}$-specific IFN- $\gamma$ production using intracellular cytokine analysis. We assessed PBMCs isolated before the commencement of ACT (pretherapy, before the first infusion), during ongoing ACT cycles (during therapy, before the third or fourth infusion), in the first 2 months after the completion of therapy (post short term [post ST]; median 1 month, range $0-2$ ), or at least 3 months after the completion of therapy (post long term [post LT]; median 6 months, range 3-12). Samples were selected based on availability and one 


\section{Table 2. Clinical characteristics of individual GBM patients}

\begin{tabular}{|c|c|c|c|c|c|c|c|c|}
\hline $\begin{array}{l}\text { Patient } \\
\text { Code }\end{array}$ & Age & Sex & Histological CBM type & IDH status & $\begin{array}{l}\text { MGMT } \\
\text { Status }\end{array}$ & CXT/RXT before ACT & $\begin{array}{l}\text { Treatment } \\
\text { during ACT }\end{array}$ & $\begin{array}{c}\text { Treatment } \\
\text { following ACT }\end{array}$ \\
\hline 2105-01 & 41 & M & Temporal & WT & U & XRT/TMZ & TMZ, Surgery & Nil \\
\hline 2105-02 & 66 & M & Parieto-occipital & WT & U & XRT/TMZ & TMZ, Avastin & Nil \\
\hline 2105-03 & 55 & M & Frontal/Temporal & WT & U & XRT/TMZ & TMZ & TMZ \\
\hline 2105-04 & 75 & M & Parieto-occipital & WT & M & XRT/TMZ & TMZ & TMZ, Avastin \\
\hline 2105-05 & 64 & $\mathrm{~F}$ & Parietal & WT & U & XRT/TMZ & Nil & Nil \\
\hline 2105-06 & 56 & M & Occipital & WT & U & XRT/TMZ & TMZ, Avastin, Lomustine & Nil \\
\hline 2105-07 & 51 & $\mathrm{~F}$ & Temporal & WT & U & XRT/TMZ & Not applicable & Nil \\
\hline 2105-08 & 69 & $\mathrm{~F}$ & Temporal & WT & M & XRT/TMZ & TMZ & TMZ \\
\hline 2105-09 & 40 & $M$ & Temporal & WT & U & XRT/TMZ & $\mathrm{Nil}$ & Nil \\
\hline $2105-10$ & 74 & M & Frontal & WT & M & XRT/TMZ & TMZ, Avastin & Avastin \\
\hline $2105-11$ & 64 & $\mathrm{~F}$ & Parieto-occipital & WT & U & XRT/TMZ & TMZ & TMZ Avastin, XRT \\
\hline $2105-12$ & 67 & $\mathrm{~F}$ & Parietal & WT & M & XRT/TMZ & Not applicable & Not applicable \\
\hline $2105-13$ & 57 & $\mathrm{~F}$ & Parietal & WT & M & XRT/TMZ & TMZ & TMZ, Avastin \\
\hline 2105-14 & 32 & $\mathrm{~F}$ & Parietal & Mutant & U & XRT/TMZ & TMZ & Surgery, TMZ \\
\hline 2105-15 & 63 & $\mathrm{~F}$ & Parietal & WT & M & XRT/TMZ & TMZ & TMZ, Avastin, XRT \\
\hline 2105-16 & 46 & M & Frontal & WT & M & XRT/TMZ, TMZ & Nil & Nil \\
\hline $2105-17$ & 61 & M & Temporal & WT & U & XRT/TMZ & Nil & Surgery, TMZ \\
\hline 2105-18 & 50 & M & Frontal & WT & U & XRT/TMZ & TMZ & TMZ \\
\hline 2105-19 & 71 & M & Frontal & WT & U & XRT/TMZ & Not applicable & Not applicable \\
\hline $2105-20$ & 45 & M & Frontal & WT & M & XRT/TMZ & TMZ & Surgery, TMZ \\
\hline $2105-21$ & 61 & M & Parieto-temporal & WT & M & XRT/TMZ & TMZ & Surgery, TMZ, Avastin \\
\hline $2105-22$ & 64 & M & Frontal & WT & U & XRT/TMZ & TMZ, Avastin & TMZ, Avastin \\
\hline $2105-23$ & 33 & M & Temporal & Mutant & U & XRT/TMZ & TMZ & $\mathrm{TMZ}$ \\
\hline $2105-24$ & 60 & M & Occipital & WT & U & XRT/TMZ & TMZ & Surgery, TMZ \\
\hline $2105-25$ & 53 & M & Thalamic & WT & U & XRT/TMZ & TMZ & TMZ, Avastin \\
\hline $2105-26$ & 65 & $\mathrm{~F}$ & Frontal & WT & U & XRT/TMZ & TMZ & TMZ \\
\hline $2105-27$ & 38 & $\mathrm{~F}$ & Frontal & WT & M & XRT/TMZ & TMZ & Nil \\
\hline $2105-28$ & 41 & M & Parieto-occipital & Mutant & M & XRT/TMZ & TMZ & TMZ \\
\hline
\end{tabular}

$\mathrm{TMZ}$, temozolomide; $\mathrm{XRT}$, radiation.

sample was used for each time point. The frequency of CMV-specific IFN $-\gamma^{+} \mathrm{CD}^{+}$and IFN $-\gamma^{+} \mathrm{CD} 4^{+} \mathrm{T}$ cells was determined as a proportion of total viable lymphocytes. At all time points following ACT, there was a significant increase in the frequency of CMV-specific $\mathrm{T}$ cells in PBMCs (Figure 5A), with a median CMV-specific response of $0.51 \%$ before therapy, $0.72 \%$ during therapy, $0.80 \%$ post $\mathrm{ST}$, and $1.0 \%$ post LT. We also assessed changes in the structural composition of the CMV-specific immune response in patients following CMV-specific ACT. To do this, we expanded CMV-specific T cells from PBMCs at different time points and assessed their reactivity against the peptide epitopes recognized by the autologous ACT product and HLA-matched to the patient (Supplemental Tables 1 and 2). Representative analyses from 6 patients are shown in Figure
5B. The remaining patients who were analyzed are shown in Supplemental Figure 1. While there was little change in the dynamics of the CMV-specific T cell response following ACT for some patients (represented by patient 2105-15), in the majority of patients we noted changes in the immunodominance hierarchy of CMV epitopespecific $\mathrm{T}$ cell responses that included the expansion of $\mathrm{T}$ cells against previously subdominant epitopes.

To further explore changes in the immune landscape following CMV-specific ACT, we next investigated the potential for epitope spreading by assessing $\mathrm{T}$ cell immunity to a panel of common tumor-associated antigens (TAAs) (Supplemental Table 3) that have been previously reported to be expressed in GBM (25-31). To assess immunity against these common TAAs, 


\section{Table 3. Adoptive cellular therapy product characteristics and patient survival analysis at completion of follow-up}

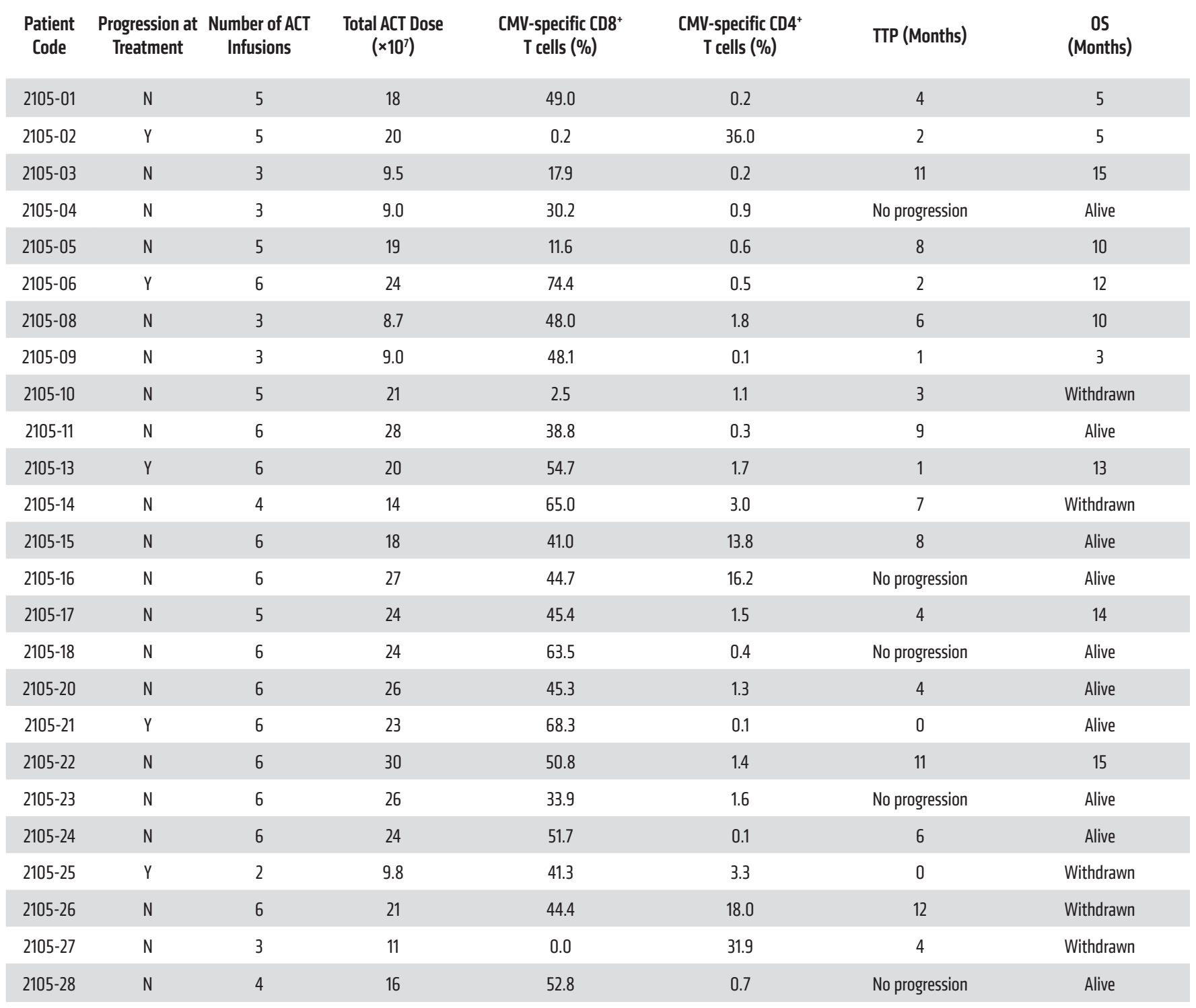

${ }^{A}$ Adverse event terms and grades based on the Common Terminology Criteria for Adverse Events version 4.0. ${ }^{\mathrm{B}}$ Number of patients experiencing each adverse event at the specified grade. All events had an association with the investigational product of "unlikely to be related." 'Occurred on 2 occasions for this patient. ${ }^{\circ}$ Occurred on 3 occasions for this patient.

we generated 3 pools of overlapping peptides (Supplemental Table 3) and stimulated PBMCs from trial participants with each individual TAA pool. Stimulated PBMCs were cultured for 2 weeks in the presence of IL-2, and then the expanded T cells were assessed for antigen specificity using an intracellular cytokine assay. Nineteen of the 25 screened patients displayed strong T cell reactivity against at least 1 of the 3 TAA pools, indicative of the priming of $T$ cell responses against these antigens in trial participants (Figure 6, A and B). While these TAA-specific responses remained stable in a large proportion of the patient cohort, we observed improved $\mathrm{T}$ cell reactivity in 9 patients following ACT. In some instances, this constituted an increase in the preexisting response (patients 2105-03, 2105-10, 2105-15, and 2105-28) while in others (patients 2105-06 and 2105-14) we observed the emergence of responses against a different TAA pool. These observations suggest that in addition to the impact on CMVspecific peripheral immunity in patients with GBM, CMV-specific ACT may demonstrate an additional bystander impact leading to additional $\mathrm{T}$ cell responses through epitope spreading.

Long-term clinical outcome of ACT in patients with primary $G B M$. To assess the potential long-term impact of CMV-specific ACT in our cohort of patients with primary GBM, we performed a follow-up survival analysis from the time of diagnosis. Median PFS in the 25 treated patients was 10 months (range 2-65 months; Figure 7A) and median OS was 21 months (range 5-65 months) following diagnosis (Figure 7B). To assess OS and PFS in patients 
Table 4. Adverse events with a relationship to the investigational product of greater than "not related"

\begin{tabular}{|c|c|}
\hline Adverse Event Term ${ }^{A}$ & No. Patients ${ }^{B}$ \\
\hline $\begin{array}{l}\text { Grade } 1 \text { events } \\
\text { Upper limb weakness } \\
\text { Headache } \\
\text { Fatigue } \\
\text { Bradycardia } \\
\text { Hypertension } \\
\text { Hypotension }\end{array}$ & $\begin{array}{l}1 \\
1 \\
1 \\
1 \\
1 \\
1\end{array}$ \\
\hline $\begin{array}{l}\text { Grade } 2 \text { events } \\
\text { Ataxia } \\
\text { Hemispatial neglect } \\
\text { Cognitive disturbance } \\
\text { Upper and lower limb weakness } \\
\text { Dexterity } \\
\text { Fatigue } \\
\text { Memory impairment }\end{array}$ & $\begin{array}{l}2 \\
2 \\
1 \\
2 \\
1 \\
1 \\
1\end{array}$ \\
\hline $\begin{array}{l}\text { Grade } 3 \text { events } \\
\text { Seizure } \\
\text { Ataxia } \\
\text { Dehydration } \\
\text { Cognitive disturbance } \\
\text { Upper and lower limb weakness } \\
\text { Right-sided hemianopia } \\
\text { Headache } \\
\text { Psychosis } \\
\text { Subdural hemorrhage } \\
\text { Dysphasia } \\
\text { Fall resulting in injury to head } \\
\text { Depressed level of consciousness } \\
\text { Thromboembolic event }\end{array}$ & $\begin{array}{c}3 \\
2 \\
2 \\
2 \\
1 \\
1 \\
1 \\
1 \\
1 \\
1 \\
1 \\
1 \\
1\end{array}$ \\
\hline $\begin{array}{l}\text { Grade } 5 \text { event } \\
\quad \text { Headache (increasing intensity) }\end{array}$ & 1 \\
\hline \multicolumn{2}{|c|}{$\begin{array}{l}{ }^{A} \text { Adverse event terms and grades based on the Common Terminology Criteria } \\
\text { for Adverse Events version 4.0. }{ }^{B} \text { Number of patients experiencing each } \\
\text { adverse event at the specified grade. All events had an association with the } \\
\text { investigational product of "unlikely to be related." 'Occurred on } 2 \text { occasions } \\
\text { for this patient. }{ }^{D} \text { Occurred on } 3 \text { occasions for this patient. }\end{array}$} \\
\hline
\end{tabular}

treated with ACT before evidence of disease progression, we stratified patients based on disease status at the time of the first infusion (Figure 7, C and D). Patients treated with ACT before recurrence $(n=20)$ showed significantly improved OS compared with those who progressed $(n=5)$ before ACT (23 months, range 7-65 vs. 14 months, range $5-19 ; P=0.018$ ). The 20 patients treated before progression had a median PFS of 10 months (range 4-65 months). The PFS and OS of the intent-to-treat cohort, all treated patients, and patients treated preemptively before progression at 6,12 , and 24 months are listed in Table 5.

Association of ACT gene expression profile with long-term survival following CMV-specific ACT. Finally, to assess the potential impact of ACT product quality attributes on patient survival, we investigated whether there was any association between $\mathrm{T}$ cell gene expression profiles (assessed using the NanoString nCounter gene expression platform) and the OS of patients treated with these products. We performed an unsupervised hierarchical clustering of the ACT product expression profiles, which revealed 4 distinct patient clusters, C1 to C4 (Supplemental

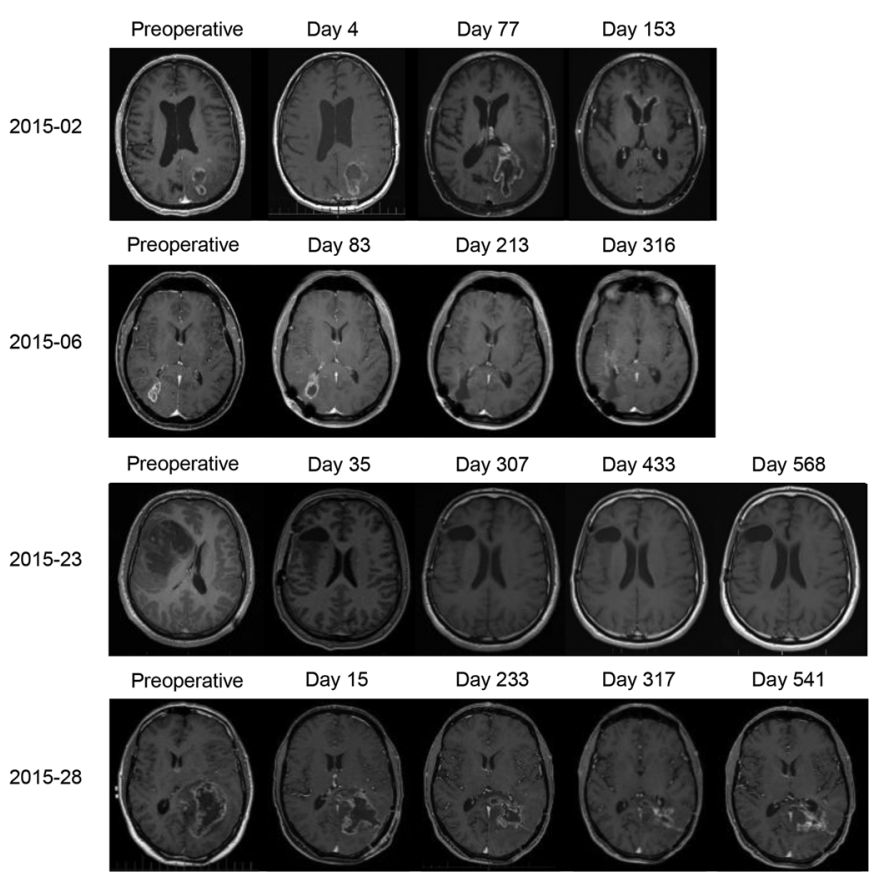

Figure 3. Representative MRI scans following CMV-specific ACT. Scans from 4 patients including 2105-02 and 2105-06 who progressed before ACT and patients 2105-23 and 2105-28, who remained disease free during the follow-up period.

Figure 2). Of these, $\mathrm{C} 1$ and $\mathrm{C} 3$ had 10 and 7 patients, respectively, and were deemed large enough for survival comparisons. Differential expression analysis between the 2 clusters identified 46 genes $(P<0.05$ and fold change $[\mathrm{FC}]>1.5$; Figure $8 \mathrm{~A})$. For example, higher expression of the key transcription factor eomesodermin (EOMES) was strongly associated with C3. Other genes associated with this cluster included $\mathrm{T}$ cell signaling and immune regulation molecules cystatin $\mathrm{F}$ (CST7), killer cell lectin-like receptor subfamily $\mathrm{D}$, member 1 (KLRD1), killer cell lectin-like receptor subfamily $\mathrm{D}$, member 1 (KLRG1), protein tyrosine phosphatase, nonreceptor type 6 (PTPN6), homing markers such as integrin $\alpha \mathrm{L}$ chain (ITGAL/CD11a), and effector molecule granzyme H (GZMH) (Figure 8B). Pathway enrichment analysis highlighted several differentially activated pathways between the 2 clusters - e.g., cell cycle and proliferation-related pathways were significantly enriched in C1, whereas pathways related to natural killer cell-mediated toxicity and immunity were enriched in C3 (Supplemental Figure 3). Conversely, T cells from patients in $\mathrm{C} 1$ showed overexpression of IL-23, SELL, and NYB (Figure 8B). Interestingly, these 2 clusters differed significantly in their OS based on 72 months' follow-up from diagnosis $(P=0.026$; hazard ratio for $\mathrm{C} 1$ vs. $\mathrm{C} 3,8.5 ; P=0.05$; Figure $8 \mathrm{C})$, with $\mathrm{C} 1$ showing considerably poorer OS (median OS 21 months) compared with C3 (median OS not reached). The observed difference was maintained after removing the 4 patients who had tumor progression before the first ACT infusion $(P=0.024$; Figure 8D), and also upon including $M G M T$ methylation status and $I D H 1$ mutation status in a multivariate survival (Figure 8, E and F) and hazard-ratio (Supplemental Figure 4) analysis. In particular, C3 patients with MGMT methylation and/or 


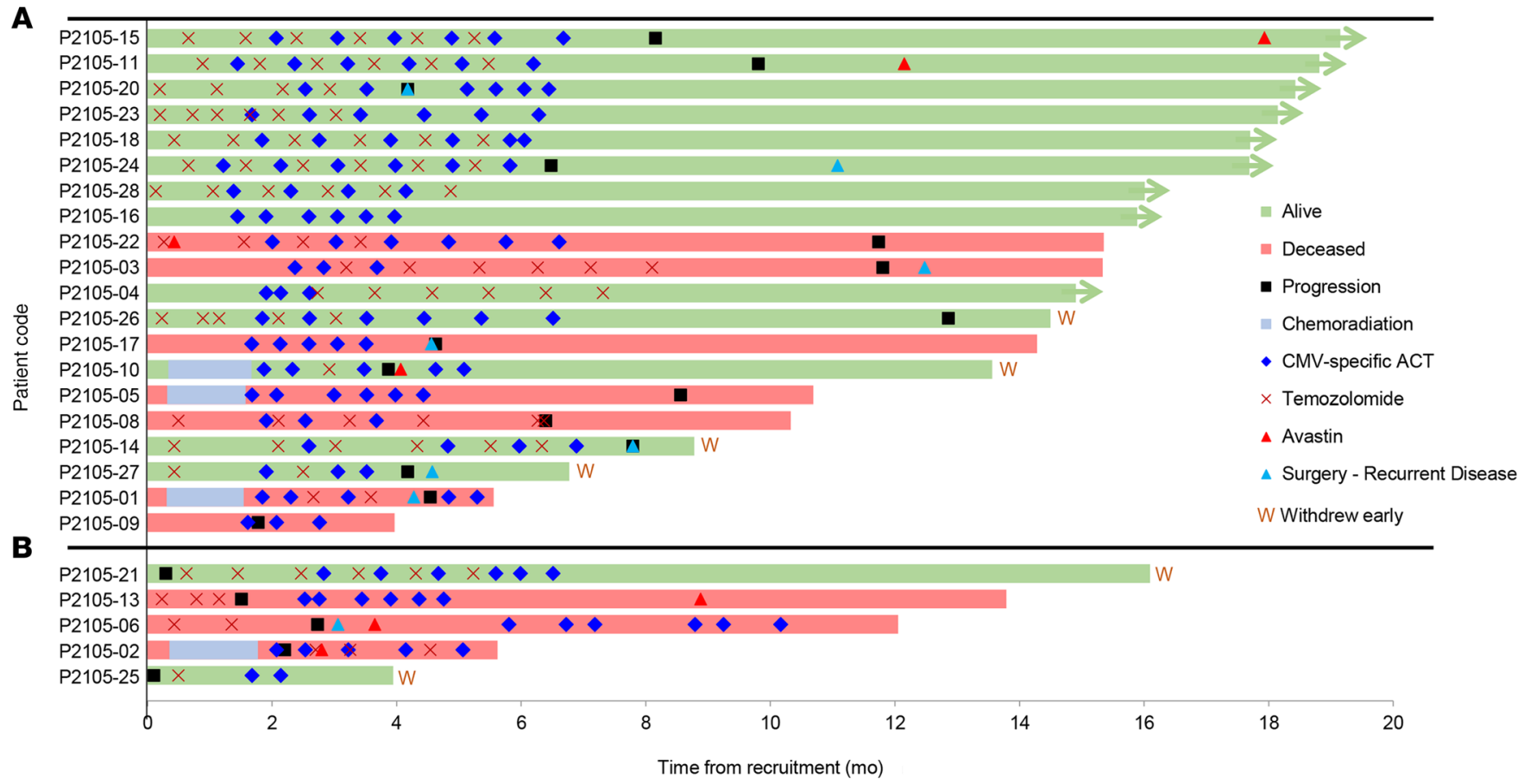

Figure 4. Swimmer plot of clinical course of patients following recruitment. Plot shows timing of ACT infusions and (where relevant), tumor progression, chemoradiation, temozolomide, Avastin, surgery, withdrawal or death for patients treated with ACT. Panel A shows clinical course of patients who were treated with ACT before recurrence, while panel $\mathbf{B}$ shows clinical course of patients who were treated after recurrence. The key within the plot describes all symbols and color coding.
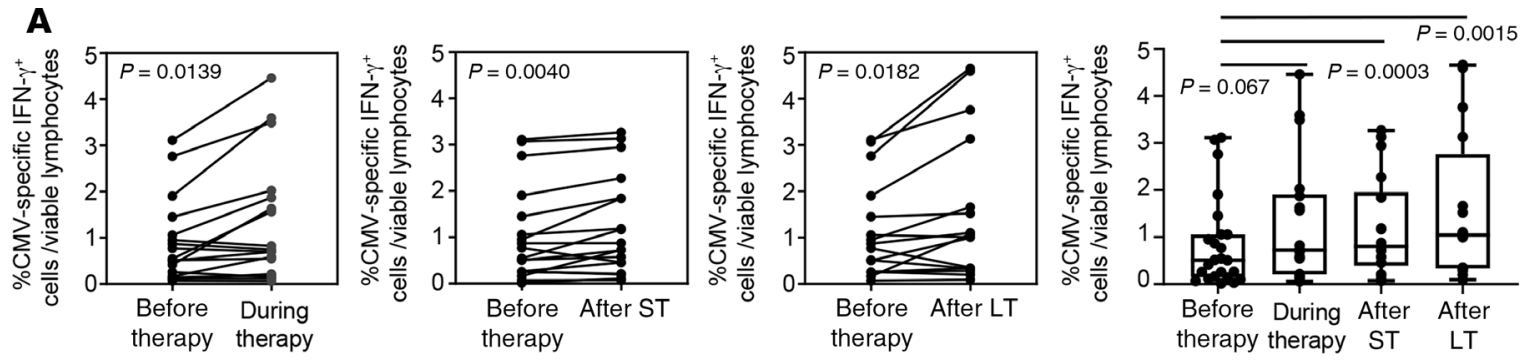

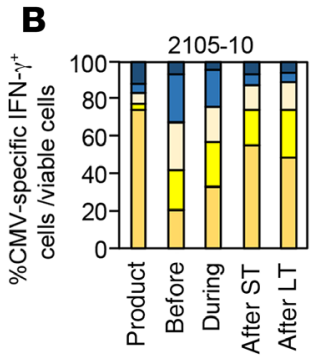

口VTE םYSE वRPH $\square T P R$

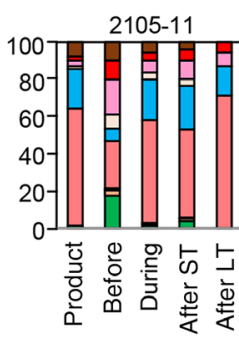

口YAY $\square S E H \square Q E F$ 口PLK $\square$ NLV $\square E E A$ aDYS $\triangle C M L \square C E D$

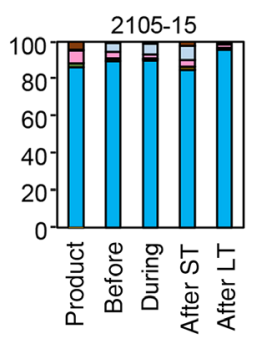

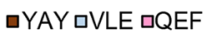
口PLK $\square D Y S \square D E L$ $\square N L V$

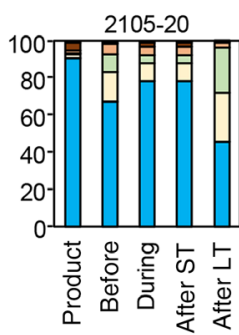

口YAY $S E H \square E H P$ 口HERםELR $\square$ NLV

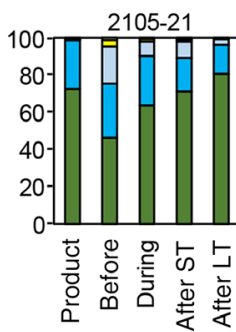

aELK $\square$ RPH $\square$ VLE $\square$ NLV $\square$ QIK

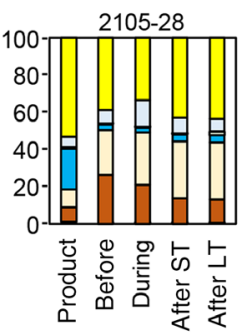

口RPHםQYDaQIK $\square$ NLV $\square E L R$ 口CRV $\square C P S$

Figure 5. CMV-specific immunological monitoring following ACT. (A) PBMCs isolated from patients pre-ACT ( $n=18)$, during ACT $(n=18)$, and either short-term (ST) ( $n=18)$ or long-term (LT) ( $n=18)$ after ACT were assessed for IFN- $\gamma$-producing CMV-specific T cells following stimulation with the CMV peptide pool used to generate the ACT products. Samples were selected based on sample availability and at the time point nearest to the following: first infusion day (pre-ACT), mid-point of infusion (during ACT), a median of 1 month following completion (short-term), and a median of 6 months following completion (long-term). Data represent paired patient analyses of the frequency of CMV-specific IFN- $\gamma$-producing cells as a proportion of total lymphocytes. Wilcoxon's matched-pairs signedrank tests were used to determine significant differences in paired analysis. The box-and-whisker plot represents a summary of all patient responses. Statistical analysis was performed using mixed-effects analysis with Dunnett's multiple-comparisons test. Time points were considered statistically significant when $P<$ 0.05. (B) PBMCs isolated from patients at different time points during the clinical trial were stimulated for 14 days with the CMV peptide pool, and then assessed for reactivity against individual HLA-matched peptides contained in the pool. Representative data from 6 donors show the proportion of IFN- $\gamma$-producing peptide-specific T cells as a proportion of total CMV-specific T cells. Additional patient data are provided in Supplemental Figure 1. 
A

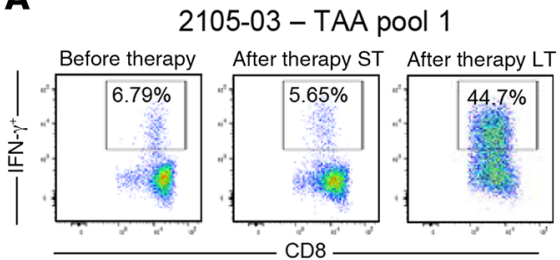

2105-17 - TAA pool 1

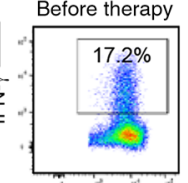



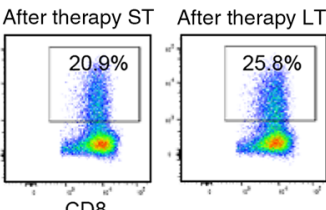

$\mathrm{CD} 8$
2105-15 - TAA pool 2

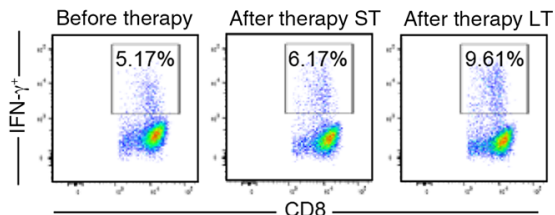

B

GBM patient code

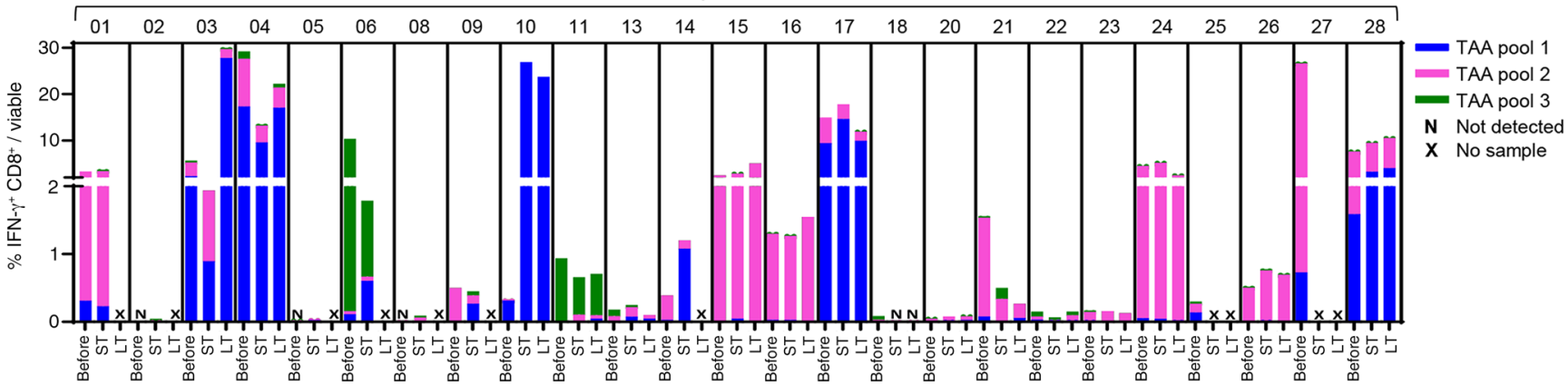

Figure 6. GBM-associated TAA-specific immunological monitoring following ACT. PBMCs isolated from patients at different time points during the clinical trial were stimulated with pools of GBM-associated TAAs, and then cultured for 14 days in the presence of IL-2. T cell cultures were then assessed for reactivity against the peptide pools using an intracellular cytokine assay. (A) Representative analysis of T cell responses from 3 different patients. (B) Data represent the frequency of IFN $-\gamma^{+}$CD8 ${ }^{+}$T cells in response to each peptide pool. Patients were assessed at 3 time points depending on the availability of samples. Pretherapy $(n=25)$ was before the first infusion, posttherapy ST $(n=23)$ and posttherapy LT $(n=20)$.

WT IDH1 performed considerably better than the corresponding $\mathrm{C} 1$ patients. These observations strongly support an association between transcriptional profiles of ACT products and OS of patients. The 3 remaining patients - P2105-27 (C2), P2105-
09 (C4), and P2105-17 (C4) - had the poorest outcomes (all deceased within 21 months of diagnosis) and the ACT product profiles of these patients clustered separately from C1 and C3 (Supplemental Figure 2).
A
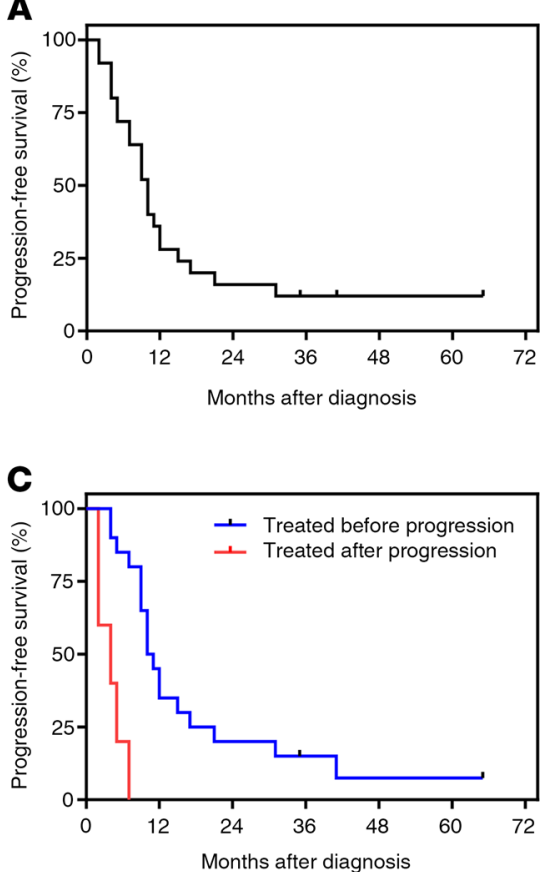

B

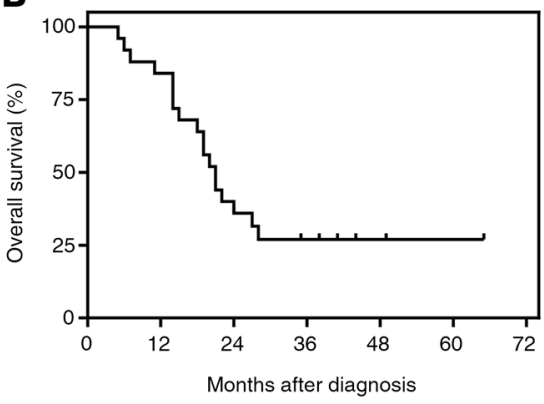

D

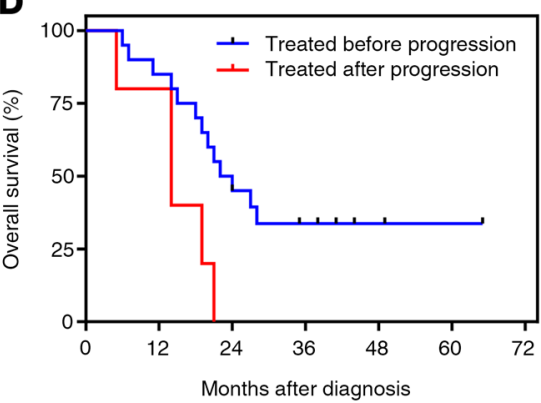

Figure 7. Long-term progression-free and overall survival of patients with GBM following CMV-specific ACT. Progression-free (A) and overall (B) survival analysis was performed for all 25 patients treated with CMV-specific ACT. Patients were stratified based on their time of progression, either before therapy $(n=5)$ or after therapy $(n=20)$, and progression-free $(\mathbf{C})$ and overall survival $(\mathbf{D})$ were determined. All survival calculations were performed from the time of initial diagnosis. 
Table 5. Longer-term survival characteristics in all patients from primary diagnosis

\begin{tabular}{cccccc} 
& & $\begin{array}{c}6 \text { months } \\
(\%)\end{array}$ & $\begin{array}{c}12 \text { months } \\
(\%)\end{array}$ & $\begin{array}{c}\mathbf{2 4} \text { months } \\
(\%)\end{array}$ & Median \\
\hline $\begin{array}{c}\text { Intent to treat } \\
(\boldsymbol{n}=\mathbf{2 8 )}\end{array}$ & PFS & 71 & 32 & 17 & 10 \\
\hline Treated & PFS & 89 & 82 & 36 & 21 \\
$(\boldsymbol{n}=\mathbf{2 5})$ & OS & 92 & 28 & 16 & 10 \\
Preemptive & PFS & 80 & 84 & 36 & 21 \\
$(\boldsymbol{n}=\mathbf{2 0})$ & OS & 95 & 85 & 20 & 10 \\
\hline $\begin{array}{c}\text { Therapeutic } \\
(\boldsymbol{n}=\mathbf{5})\end{array}$ & PFS & 20 & 0 & 45 & 23 \\
& OS & 80 & 80 & 0 & 4 \\
& & & & & 14
\end{tabular}

\section{Discussion}

Despite the progress made in treating many cancer types in recent decades, significant improvement in the OS of patients with GBM since the introduction of temozolomide has remained elusive (3235). These observations have recently been reinforced by disappointing results using PD-1 checkpoint blockade to treat GBM (9, 12). Nevertheless, evidence from a number of studies has demonstrated the potential of exploiting the immune system to augment OS in patients with GBM $(14,36-38)$. In the current single-arm open-label study, we have harnessed the association of CMV with GBM tissue and used CMV-specific ACT to directly target this disease. We have demonstrated the strong safety profile of this therapy, accompanied by potential for favorable outcomes in patients following primary tumor resection. In a cohort of 25 patients treated with ACT, we demonstrated a median PFS of 10 months and OS of 21 months following diagnosis, which compares favorably to the standard-of-care outcomes of 5.9 to 7.3 months and 14.6 to 17.3 months, respectively $(34,39-41)$. We also demonstrated improvement in peripheral cellular immunity to CMV and TAAs expressed in GBM following ACT, which is indicative of the potential of CMV-specific ACT to modulate global immunity to GBM.

Due to the rapid progression of GBM, early immunotherapeutic intervention in addition to tumor debulking with surgery, radiotherapy, and chemotherapy is likely to provide the most effective means to augment OS in patients. We previously reported the use of autologous CMV-specific ACT in 11 patients with recurrent GBM, and recently provided an update on 4 of these patients who have demonstrated impressive OS of up to 9 years following treatment (24). However, due to the advanced stage of recurrent disease, we were unable to provide treatment for 8 patients recruited to this previous study (18). Thus, the current study was designed to assess the safety and potential efficacy of CMV-specific ACT following primary diagnosis, providing treatment before relapse, while tumor burden is low. We have demonstrated the feasibility of our current approach, evidenced by the capacity to generate cell therapy from 27 of 28 recruited patients, and the treatment of 20 patients before relapse. However, the rapid progression typical of GBM was apparent in our cohort and precluded the treatment of all patients before progression. Two patients experienced tumor progression that prohibited them receiving ACT and a further 5 received treatment after the progression of their disease. Other strategies, including "off-the-shelf" allogeneic cellular therapies, may be considered to treat patients who rapidly progress following primary treatment. Allogeneic antigen-specific ACT has been successfully used to treat virus-associated lymphomas and posttransplant infectious complications $(42,43)$.

This early intervention approach has been the focus of many immune interventions recently developed for GBM. Recent observations have shown that the application of checkpoint inhibition in the neoadjuvant setting before surgery can significantly improve outcomes compared with an adjuvant approach in recurrent GBM $(14,36)$. Similarly, recent studies have applied personalized neoantigen vaccines for patients with GBM, administered within 6 months following primary diagnosis, with median OS of 16.8 and 29 months observed in 2 separate studies $(37,38)$. CMV-targeted vaccine strategies have also recently focused on treatment following primary standard of care, with encouraging survival results (44). These observations provide promise for early immunological intervention for GBM treatment. It should be noted that, like our study, these vaccine studies were single-arm open-label trials.

Although recent results with neoadjuvant checkpoint inhibition in patients with GBM is promising, the limited mutational burden and poor $\mathrm{CD}^{+} \mathrm{T}$ cell infiltrate in GBM tissue suggests that augmentation of the $\mathrm{T}$ cell response to GBM will be critical for the successful application of immunotherapy $(45,46)$. Furthermore, this will be dependent on the selection of appropriate antigenic targets. Current chimeric antigen receptor approaches have focused on a number of potential targets, including EGFRVIII, IL-13R $\alpha 2$, and HER2, with promising early results (13, $15,47)$. In addition to CMV antigens and neoantigens, TAAs have also been investigated as targets for immunotherapy in GBM (25, $48,49)$. Interestingly, in our study we noted the potential effect of targeting CMV on the $\mathrm{T}$ cell response to other TAAs expressed in GBM. Although the impact of these changes on outcome was inconclusive, they provide evidence of a bystander effect or epitope spreading, which has been proposed to broaden the tumorspecific $\mathrm{T}$ cell response, with likely implications for long-term tumor control. Similar observations have previously been made in other settings of immunotherapy, including the use of EpsteinBarr virus-specific T cell therapy to treat B cell lymphomas (50). These observations may also provide an opportunity for dual targeting of viral and tumor antigens, to improve efficacy of immunotherapies against GBM. A recent phase I clinical trial in the GBM setting used HER2-CMV bispecific CAR T cells; these displayed a good safety profile and resulted in clinical benefit (51). Augmentation of cell therapy approaches with checkpoint inhibition could provide further benefit to clinical outcome.

Our current study provides clear evidence of the robustness of our protocol for the generation of autologous ACT from patients with GBM, and the safety and feasibility of using ACT in an adjuvant setting to preemptively treat GBM before relapse. We also provide clear evidence of the impact CMV-specific ACT can have on peripheral immunity in this cohort. In particular, we observed specific differences in transcriptional profiles at gene and pathway levels between $\mathrm{T}$ cells of clusters $\mathrm{C} 1$ and $\mathrm{C} 3$ that showed considerable difference in OS. Of particular importance, this study provides evidence that the differential effects observed following the administration of $\mathrm{T}$ cells with distinct profiles are independent of other known risk factors in GBM, including unmethylated MGMT. These transcriptional differences could 
A

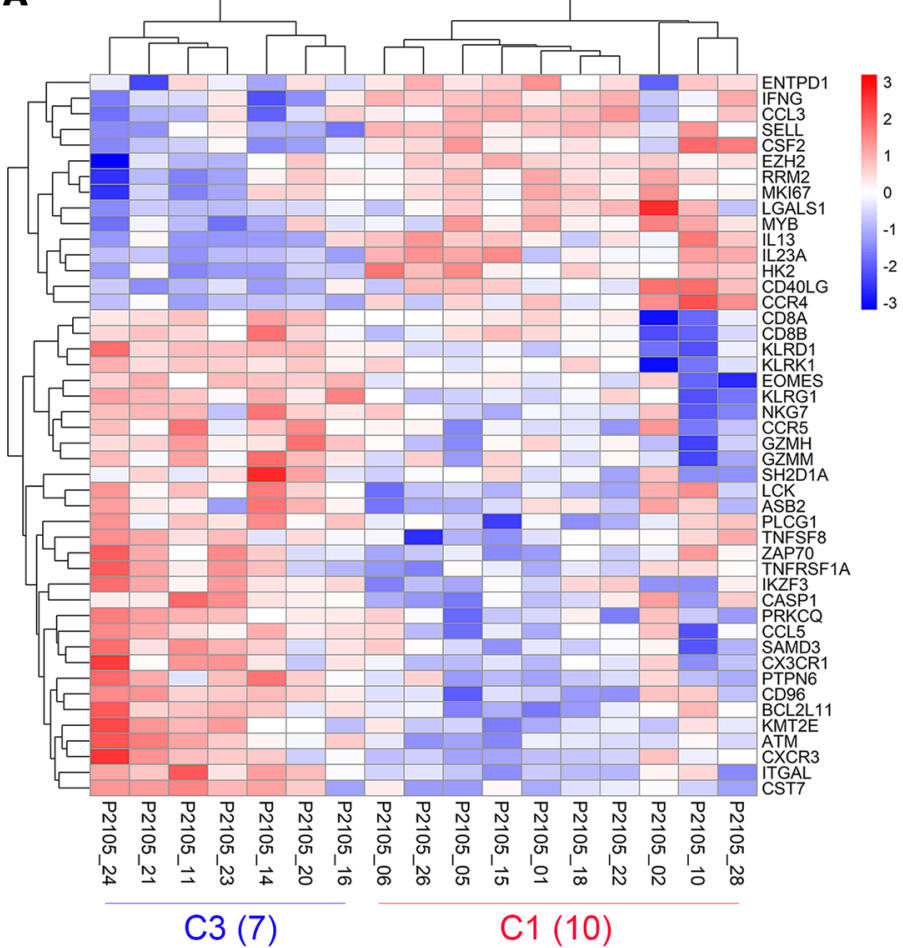
C3 (7)

C

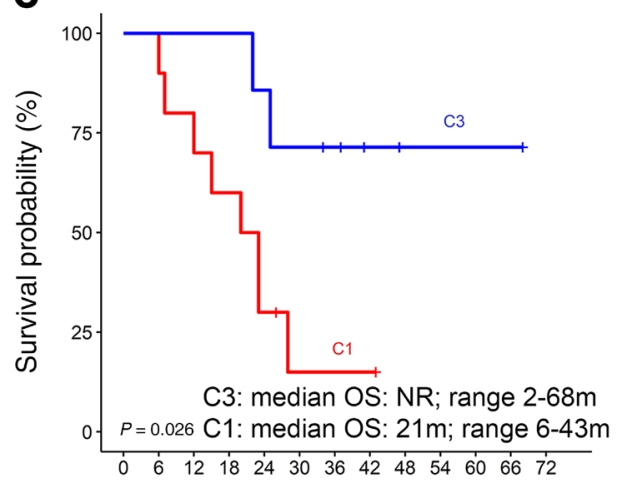

Overall survival $(\mathrm{mo})$

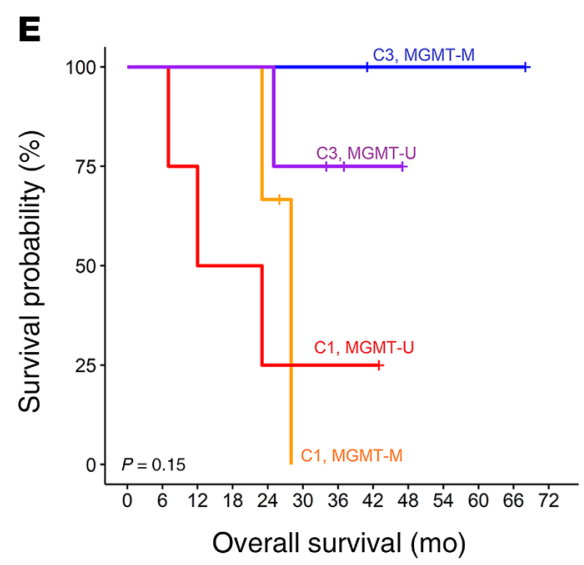

B

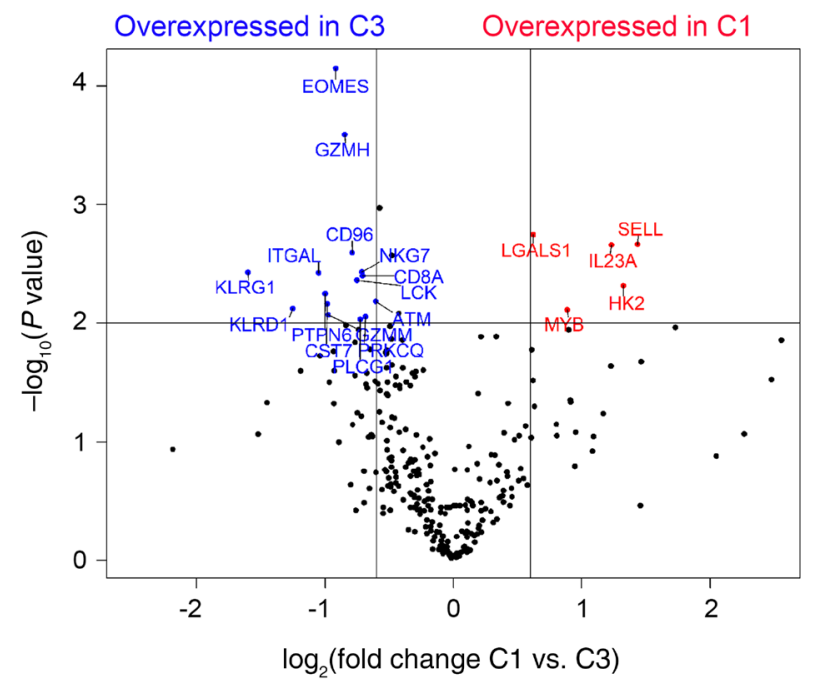

Figure 8. Gene expression profile and clustering of CMV-specific ACT products. In vitro-expanded patient CMV-specific ACT products were restimulated and IFN- $\gamma$-producing cells were used for gene expression analysis using the NanoString nCounter gene expression platform. (A) Expression heatmap of de novo patient clusters $\mathrm{C} 1$ and $\mathrm{C} 3$, identified using unsupervised hierarchical clustering of 46 differentially expressed genes $(P<$ $0.05)$. (B) Volcano plot of genes that were significantly differentially expressed between patient clusters $\mathrm{C} 1$ and $\mathrm{C} 3$. Labeled differentially expressed genes have $P<0.05$ and $\log _{2} \mathrm{FC}>0.6(\sim 1.5$ FC). Overall survival rates based on 72 months of follow-up from diagnosis of (C) all patients in clusters C1 and C3 and (D) patients that had no progression of disease before their first ACT infusion. C1 vs. C3: hazard ratio $=8.5(P=0.05)$; difference in mean survival using restricted mean survival time $=14.12$ months $(P=0.001)$. Multivariate overall survival analysis was undertaken by incorporating MGMT methylation status (E) and IDH1 mutation status (F). NR, not recorded. be leveraged to functionally manipulate specific pathways and develop $\mathrm{T}$ cells that are more effective in treating GBM. Overall, the data presented here must be interpreted with caution and 2 major limitations should be borne in mind. First, observations from this study need to be reproduced in larger controlled trials conducted concurrently at multiple clinical centers. Second, bias in the selection of patients enrolled in this study may have influenced the clinical outcome observed in the patients. In spite 
of these limitations, this study provides the impetus to develop combination or bispecific approaches targeting CMV and TAAs to further enhance the efficacy of ACT for GBM.

\section{Methods}

Patient recruitment and study design. Patients were eligible for the study if they had histological diagnosis of primary GBM (WHO grade IV), Eastern Cooperative Oncology Group performance status of 0 , 1 , or 2, life expectancy of greater than 6 months, and were CMVseropositive. The detection of CMV antigens in tumor tissue was not assessed. Patients included in the study were HLA typed (Supplemental Table 1) and received up to 6 intravenous infusions of in vitro-expanded T cells at a dose of $2 \times 10^{7}$ cells $/ \mathrm{m}^{2}$ body surface area every 2 to 4 weeks. Patients continued standard-of-care treatment during the course of immunotherapy. Where possible, administration of ACT was scheduled to fall between chemotherapy treatment weeks to avoid concurrent infusions. Each participant was monitored for safety, disease progression, and immune reconstitution for 1 year after the completion of ACT. Long-term survival analysis was performed for up to 65 months following diagnosis.

Manufacture of CMV-specific T cells for ACT. The protocol used to manufacture CMV-specific T cells for ACT has been described recently (52). Briefly, PBMCs were harvested from peripheral blood within 24 hours of venesection. One-third of the PBMCs were then incubated with a clinical-grade custom CMV peptide pool for 1 hour, washed, mixed with the remaining PBMCs (JPT Technologies; Supplemental Table 2), and then seeded in G-Rex10 culture flasks (Wilson Wolf) at a density of between $2 \times 10^{6}$ and $5 \times 10^{6}$ cells $/ \mathrm{cm}^{2}$. Cells were grown in culture medium containing recombinant IL-21 from day 0 , with recombinant IL-2 added every 2 to 3 days thereafter. On day 14, ACT products were harvested and frozen in $1 \mathrm{~mL}$ single-dose aliquots in Albumex 4 (CSL Behring) containing 10\% dimethyl sulfoxide (WAK-Chemie Medical). ACT products were phenotypically and functionally characterized using Multitest 6-color TBNK Reagent (BD Biosciences) and intracellular cytokine assay, and microbial testing was performed as previously described (52).

Intracellular cytokine assay. ACT products, PBMCs, or laboratory-cultured $\mathrm{T}$ cells were stimulated with the custom CMV peptide pool, individual HLA-matched epitopes in the CMV peptide pool (Supplemental Table 2), or TAA pools (Supplemental Table 3). Cells were cultured for 4 hours in the presence of GolgiPlug and GolgiStop (BD Biosciences) and anti-CD107a-FITC (BD Biosciences, clone BerACT8), then washed and stained with anti-CD8-PerCP-Cy5.5 (eBioscience, clone RPA-T8) and anti-CD4-PE-Cy7 or anti-CD4-Pacific Blue (BD Biosciences, clone RPA-T4), fixed and permeabilized with Cytofix/Cytoperm (BD Biosciences), washed again and stained with anti-IFN- $\gamma$-Alexa Fluor 700 (clone B27), anti-IL-2-PE (clone MQ117H12), and anti-TNF-APC (clone Mab11) (all from BD Biosciences). Cells were washed, then resuspended in PBS and acquired using a BD LSRFortessa with FACSDiva software (BD Biosciences). Postacquisition analysis was performed using FlowJo software (Tree Star).

Gene expression profiling of CMV-specific ACT products. To enrich CMV-specific $\mathrm{CD}^{+}$and $\mathrm{CD} 4^{+} \mathrm{T}$ cells from the ACT products, the IFN- $\gamma$ Secretion Assay Detection Kit (Miltenyi Biotec) was used according to the manufacturer's instructions. Briefly, ACT products were thawed, then $10 \%$ of the cells were coated with a pool of CMV peptides (Supplemental Table 2) for 1 hour. These stimulators were washed and incubated with the remaining ACT product cells for 3 hours. Subsequently, the cells were washed with cold buffer ( $2 \mathrm{mM}$ EDTA and $0.5 \%$ bovine serum albumin in PBS) and labeled with IFN- $\gamma$ catch reagent on ice. ACT products were then resuspended in warmed culture medium and incubated for 45 minutes at $37^{\circ} \mathrm{C}$, using a rotor wheel on low speed to maintain the cells in suspension. Products were washed again with cold buffer, and then labeled on ice with FITC-conjugated IFN- $\gamma$ detection antibody, anti-CD8PerCP-Cy5.5 (eBioscience, clone RPA-T8), anti-CD4-Pacific Blue (BioLegend, clone RPA-T4), and LIVE/DEAD Fixable Near-IR Dead Cell Stain (Molecular Probes). $\mathrm{CD}^{+}$and $\mathrm{CD} 8^{+}$IFN- $\gamma$-secreting cells were sorted using a FACSAria III (BD Biosciences) and RNA was extracted from sorted cells (RNeasy Mini Kit, QIAGEN). Gene expression analysis was conducted using the NanoString nCounter gene expression platform (NanoString Technologies) according to the manufacturer's instructions. A custom code set consisting of genes involved in T cell biology, immune regulation, and immune cellular markers was used. The samples were scanned at maximum scan resolution on the nCounter Digital Analyzer and gene expression data normalized by housekeeping gene expression to identify differentially expressed genes. De novo patient clusters were identified using unsupervised hierarchical clustering of NanoString expression profiles of ACT products that were infused into trial participants. Of the 25 treated patients, sufficient CMV-specific ACT product was available for NanoString profiling of 20 products. After removing genes with low expression, evaluation of the number of clusters $(k=2-5)$ resulted in $k=4$ patient clusters, $\mathrm{C} 1$ to $\mathrm{C} 4$, with the best intracluster homogeneity (minimum sum of squared error of Euclidean distances). Of these clusters, C1 and C3, which contained 17 of the 20 patients, were used for survival and differential expression analysis.

Statistics. OS and PFS were calculated from the date of recruitment to the date of radiologically documented progressive disease or the date of death. Patients who were alive and without evidence of progressive disease were censored at their last follow-up. Prism 8 software (GraphPad) was used to generate Kaplan-Meier plots and calculate median survival and percentage survival at different intervals. Mixed-effects analysis with Dunnett's multiple-comparisons test was performed in Prism 8 software and used to assess the significance of changes in CMV-specific T cell frequency in PBMCs following ACT. Statistical significance was defined as $P$ less than 0.05 (2-sided).

For clustering analysis, OS was calculated from the date of diagnosis to the most recent follow-up (December 2019), and OS curves with log-rank, $t$ test, $P$ value, and median survival were plotted for patients in clusters C1 $(n=10)$ and C3 $(n=7)$ using the ggsurvplot package in R v3.2. A second set of OS curves was plotted after excluding the 4 patients in these clusters who had disease progression before their first ACT infusion. ACT product genes that were differentially expressed between the 2 largest patient clusters, C1 and C3, were computed using a $t$ test followed by Bonferroni's correction. A volcano plot using the corrected $t$ test $P$ values and $\log _{2}$ fold changes was plotted using $\mathrm{R}$, to highlight the most significantly differentially expressed genes $\left(P<0.05\right.$ and $\log _{2} \mathrm{FC}>0.6$, corresponding with an approximately 1.5 -fold change in expression levels) between the 2 patient clusters. Pathways that were differentially enriched between clusters C1 and C3 were computed based on the differentially expressed genes between $\mathrm{C} 1$ and $\mathrm{C} 3$, using the InnateDB platform (https://www.innatedb.com). In addition, the pathways that were 
enriched within each of these clusters C1 and C3 were computed using the full NanoString panel of genes that were overexpressed in these clusters (i.e., genes that showed higher median expression in C1 or C3 compared with their median expression across all patients).

Study approval. This prospective study was conducted according to the principles of the Declaration of Helsinki and approved by the QIMR Berghofer Medical Research Institute Human Research Ethics Committee and UnitingCare Health Human Research Ethics Committee. This study was registered under the Australian New Zealand Clinical Trial Registry (ACTRN12615000656538), and all patients provided written informed consent before commencing participation in the trial.

\section{Author contributions}

RK, CS, and DW designed this study. RK, CS, DW, KEL, JPM, GRA, and SS contributed to drafting of the manuscript. CS, KEL,
JPM, GRA, SR, PC, AP, LB, LLT, and SS conducted experimental studies and analyzed the data. MAN, BM, and KKM were responsible for clinical trial monitoring, ethics approval, and patient recruitment. DW, MAN, BM, and KKM also contributed to clinical data analysis.

\section{Acknowledgments}

This study was supported by a Program Grant funding by the National Health and Medical Research Council of Australia (grant APP1132519). In addition, this study was also supported through generous donations by various public donors.

Address correspondence to: Rajiv Khanna, QIMR Berghofer Medical Research Institute, 300 Herston Road, Herston, Queensland, Australia. Phone: 61.7.3362.395; Email: rajiv.khanna@qimr.edu.au.
1. Maus MV, Fraietta JA, Levine BL, Kalos M, Zhao $\mathrm{Y}$, June $\mathrm{CH}$. Adoptive immunotherapy for cancer or viruses. Annu Rev Immunol. 2014;32:189-225.

2. Heslop HE, et al. Long-term outcome of EBVspecific T-cell infusions to prevent or treat EBVrelated lymphoproliferative disease in transplant recipients. Blood. 2010;115(5):925-935.

3. Park JH, et al. Long-term follow-up of CD19 CAR therapy in acute lymphoblastic leukemia. $N$ Engl JMed. 2018;378(5):449-459.

4. Fuca G, Reppel L, Landoni E, Savoldo B, Dotti G. Enhancing chimeric antigen receptor T-cell efficacy in solid tumors. Clin Cancer Res. 2020;26(11):2444-2451.

5. Chia WK, et al. Adoptive T-cell transfer and chemotherapy in the first-line treatment of metastatic and/or locally recurrent nasopharyngeal carcinoma. Mol Ther. 2014;22(1):132-139.

6. Pauken KE, Wherry EJ. Overcoming T cell exhaustion in infection and cancer. Trends Immunol. 2015;36(4):265-276.

7. Stupp R, et al. Radiotherapy plus concomitant and adjuvant temozolomide for glioblastoma. N Engl JMed. 2005;352(10):987-996.

8. Cantrell JN, et al. Progress toward long-term survivors of glioblastoma. Mayo Clin Proc. 2019;94(7):1278-1286.

9. Reardon DA, et al. OS10.3 Randomized phase 3 study evaluating the efficacy and safety of nivolumab vs bevacizumab in patients with recurrent glioblastoma: CheckMate 143. Neuro Oncol.2017;19(suppl_3):iii21.

10. Lai A, et al. Phase II study of bevacizumab plus temozolomide during and after radiation therapy for patients with newly diagnosed glioblastoma multiforme. J Clin Oncol. 2011;29(2):142-148.

11. Jue TR, McDonald KL. The challenges associated with molecular targeted therapies for glioblastoma. J Neurooncol. 2016;127(3):427-434.

12. Reardon DA, et al. Phase II study of pembrolizumab or pembrolizumab plus bevacizumab for recurrent glioblastoma (rGBM) patients. J Clin Oncol. 2018;36(15 suppl):2006.

13. O'Rourke DM, et al. A single dose of peripherally infused EGFRvIII-directed CAR T cells mediates antigen loss and induces adaptive resistance in patients with recurrent glioblastoma. Sci Transl Med. 2017;9(399).
14. Cloughesy TF, et al. Neoadjuvant anti-PD-1 immunotherapy promotes a survival benefit with intratumoral and systemic immune responses in recurrent glioblastoma. Nat Med. 2019;25(3):477-486.

15. Brown CE, et al. Regression of glioblastoma after chimeric antigen receptor T-cell therapy. $N$ Engl JMed. 2016;375(26):2561-2569.

16. Reap EA, et al. Dendritic cells enhance polyfunctionality of adoptively transferred $\mathrm{T}$ cells that target cytomegalovirus in glioblastoma. Cancer Res. 2018;78(1):256-264.

17. Weathers SP, et al. Glioblastoma-mediated immune dysfunction limits CMV-specific T cells and therapeutic responses: results from a phase I/ II trial. Clin Cancer Res. 2020;26(14):3565-3577.

18. Schuessler A, et al. Autologous T-cell therapy for cytomegalovirus as a consolidative treatment for recurrent glioblastoma. Cancer Res. 2014;74(13):3466-3476.

19. Holdhoff M, et al. Absence of cytomegalovirus in glioblastoma and other high-grade gliomas by real-time PCR, immunohistochemistry, and in situ hybridization. Clin Cancer Res. 2017;23(12):3150-3157.

20. Lucas KG, Bao L, Bruggeman R, Dunham K, Specht C. The detection of CMV pp65 and IE1 in glioblastoma multiforme. J Neurooncol. 2011;103(2):231-238.

21. Cobbs CS, et al. Human cytomegalovirus infection and expression in human malignant glioma. Cancer Res. 2002;62(12):3347-3350.

22. Lamano JB, et al. Long-term glioblastoma survival following recovery from cytomegalovirus colitis: A case report. J Clin Neurosci. 2019;64:18-21.

23. Prins RM, Cloughesy TF, Liau LM. Cytomegalovirus immunity after vaccination with autologous glioblastoma lysate. NEngl JMed. 2008;359(5):539-541.

24. Walker DG, et al. Impact of pre-therapy glioblastoma multiforme microenvironment on clinical response to autologous CMV-specific T-cell therapy. Clin Transl Immunology. 2019;8(11):e01088.

25. Liu G, Ying H, Zeng G, Wheeler CJ, Black KL, Yu JS. HER-2, gp100, and MAGE-1 are expressed in human glioblastoma and recognized by cytotoxic T cells. Cancer Res. 2004;64(14):4980-4986.

26. Saikali S, et al. Expression of nine tumour antigens in a series of human glioblastoma multiforme: interest of EGFRvIII, IL-13Ralpha2, gp100 and TRP-2 for immunotherapy.

J Neurooncol. 2007;81(2):139-148.

27. Nakahara Y, Okamoto H, Mineta T, Tabuchi K. Expression of the Wilms' tumor gene product WT1 in glioblastomas and medulloblastomas. Brain Tumor Pathol. 2004;21(3):113-116.

28. Vinagre J, et al. Frequency of TERT promoter mutations in human cancers. Nat Commun. 2013;4:2185.

29. Novak Z, et al. Enzyme-linked immunosorbent assay method for detection of cytomegalovirus strain-specific antibody responses. Clin Vaccine Immunol. 2009;16(2):288-290.

30. Das A, Tan WL, Teo J, Smith DR. Expression of survivin in primary glioblastomas. JCancer Res Clin Oncol. 2002;128(6):302-306.

31. Zhang JG, et al. Antigenic profiling of glioma cells to generate allogeneic vaccines or dendritic cell-based therapeutics. Clin Cancer Res. 2007;13(2 pt 1):566-575.

32. Johnson DR, et al. Overall survival in patients with glioblastoma before and after bevacizumab approval. Curr Med Res Opin. 2018;34(5):813-820.

33. Wick W, et al. Lomustine and bevacizumab in progressive glioblastoma. $\mathrm{N} \mathrm{Engl} \mathrm{JMed.}$ 2017;377(20):1954-1963.

34. Stupp R, et al. Effects of radiotherapy with concomitant and adjuvant temozolomide versus radiotherapy alone on survival in glioblastoma in a randomised phase III study: 5-year analysis of the EORTCNCIC trial. Lancet Oncol. 2009;10(5):459-466.

35. Gilbert MR, et al. Dose-dense temozolomide for newly diagnosed glioblastoma: a randomized phase III clinical trial. JClin Oncol. 2013;31(32):4085-4091.

36. Schalper KA, et al. Neoadjuvant nivolumab modifies the tumor immune microenvironment in resectable glioblastoma. Nat Med. 2019;25(3):470-476.

37. Hilf $\mathrm{N}$, et al. Actively personalized vaccination trial for newly diagnosed glioblastoma. Nature. 2019;565(7738):240-245.

38. Keskin DB, et al. Neoantigen vaccine generates intratumoral $\mathrm{T}$ cell responses in phase Ib glioblastoma trial. Nature. 2019;565(7738):234-239.

39. Chinot OL, et al. Bevacizumab plus radiotherapytemozolomide for newly diagnosed glioblastoma. 
NEnglJMed.2014;370(8):709-722.

40. Gilbert MR, et al. A randomized trial of bevacizumab for newly diagnosed glioblastoma. $N$ Engl JMed. 2014;370(8):699-708.

41. Herrlinger U, et al. Bevacizumab plus irinotecan versus temozolomide in newly diagnosed O6-methylguanine-DNA methyltransferase nonmethylated glioblastoma: the randomized GLARIUS trial. JClin Oncol. 2016;34(14):1611-1619.

42. O'Reilly RJ, Prockop S, Hasan AN, Koehne G, Doubrovina E. Virus-specific T-cell banks for 'off the shelf' adoptive therapy of refractory infections. Bone Marrow Transplant. 2016;51(9):1163-1172.

43. Haque T, et al. Allogeneic cytotoxic T-cell therapy for EBV-positive posttransplantation lymphoproliferative disease: results of a phase 2 multicenter clinical trial. Blood. 2007;110(4):1123-1131.

44. Batich KA, et al. Long-term survival in glioblastoma with cytomegalovirus pp65-targeted vaccination. Clin Cancer Res. 2017;23(8):1898-1909.

45. Hodges TR, et al. Mutational burden, immune checkpoint expression, and mismatch repair in glioma: implications for immune checkpoint immunotherapy. Neuro Oncol. 2017;19(8):1047-1057.

46. Martincorena I, Campbell PJ. Somatic mutation in cancer and normal cells. Science. 2015;349(6255):1483-1489.

47. Ahmed N, et al. HER2-specific chimeric antigen receptor-modified virus-specific $\mathrm{T}$ cells for progressive glioblastoma: a phase 1 dose-escalation trial. JAMA Oncol. 2017;3(8):1094-1101.

48. Everson RG, et al. Efficacy of systemic adoptive transfer immunotherapy targeting NY-ESO-1 for glioblastoma. Neuro Oncol. 2016;18(3):368-378.
49. Ahmed N, et al. HER2-specific T cells target primary glioblastoma stem cells and induce regression of autologous experimental tumors. Clin Cancer Res. 2010;16(2):474-485.

50. Bollard CM, et al. Sustained complete responses in patients with lymphoma receiving autologous cytotoxic T lymphocytes targeting Epstein-Barr virus latent membrane proteins. JClin Oncol. 2014;32(8):798-808.

51. Ahmed N, et al. Autologous HER2 CMV bispecific CAR T cells are safe and demonstrate clinical benefit for glioblastoma in a phase I trial. J Immunother Cancer. 2015;3(suppl 2):O11.

52. Smith C, et al. Autologous adoptive T-cell therapy for recurrent or drug-resistant cytomegalovirus complications in solid organ transplant recipients: a single-arm open-label phase I clinical trial. Clin Infect Dis. 2019;68(4):632-640. 